\title{
Multiple Access Technologies for Beyond 3G Wireless Cellular Systems
}

\author{
R Sudheer Kumar, K Ramprasad, G Srikanth, A Pushpalatha \\ M. Tech Computational Engineering RGUKT,
}

\begin{abstract}
In this paper, some fundamental technical concepts of the main multiple access schemes for wireless mobile systems are reviewed, and a path for development of appropriate multiple access technologies for next-generation mobile networks, or so-called beyond $3 \mathrm{G}(\mathrm{B} 3 \mathrm{G})$, is established. We first review the multiple access technologies in second- and third-generation wireless cellular systems from historical and technical points of view. This review is followed by a novel formulization of the multiple access schemes through mathematical expressions that could simplify the process of development of new multiple access schemes for B3G wireless cellular systems. We then list the most important features of multiple access schemes in $3 \mathrm{G}$ mobile systems. Finally, we conclude the article by reviewing the most promising multiple access technologies for B3G mobile cellular systems.
\end{abstract}

Keywords: - Chip rate, Fading, Frequency reuse, Multiframe, Mutual Interference, RAKE diversity, Spreading rate.

\section{INTRODUCTION}

Beyond third-generation (B3G) wireless cellular systems are recognized as systems that achieve highdata-rate transmission beyond those currently provided by second- and third generation $(2 \mathrm{G} / 3 \mathrm{G})$ wireless cellular systems, and are capable of delivering multiple services to users at different locations under very diverse channel conditions. B3G systems should provide services comparable to those offered by wired networks for a variety of applications such as interactive multimedia, voice over IP, network games, and videoconferencing. The current trend is for B3G to be based on IP technology; thus, all-IP architecture design has been an important research topic in the past few years. These systems will need to support multiple services ubiquitously in different types of environments including indoor, outdoor (with low and high mobility), and up to global broadband access (satellite). Thus, they will be deployed in a heterogeneous environment of various physical access networks, both wireless and wired.

In this paper, B3G mobile systems will face at least two predominant constraints:

- Heterogeneity in the physical access network

- Change of application types from traditional low data rates into more bandwidth demanding real-time and multimedia ones.

In such situations, providing quality of service to users will become even more difficult than it is at the current time. One of the main issues involved in the development of B3G systems is the choice of multiple access (MA) technology to efficiently share the available scarce bandwidth among a large number of users. The choice of MA technique could significantly enhance or lower the service quality delivered to end users. It is known that the existing MA techniques used in 2G/3G systems, including those based on time-division (TDMA) and direct sequence code-division (DS-CDMA), and possible combinations of the two schemes are basically suitable for voice communications but not for burst data traffic, which would be the dominant portion of the traffic load in B3G systems. Therefore, the need to develop new MA techniques for B3G becomes imperative.

In this article we review the main technical concepts of the MA schemes in wireless mobile systems and try to establish a path for the development of an appropriate MA scheme for B3G through the fundamentals that govern the existing MA schemes. In the following section MA technologies are reviewed from historical and technical points of view. This is followed by a section that provides simple formulization of MA schemes through mathematical expressions that could simplify the process of developing new MA schemes for B3G wireless cellular systems. We then list the most important features of MA schemes in 3G mobile systems. Finally, we conclude this article by reviewing the most promising MA schemes for B3G mobile systems. 


\section{HISTORY}

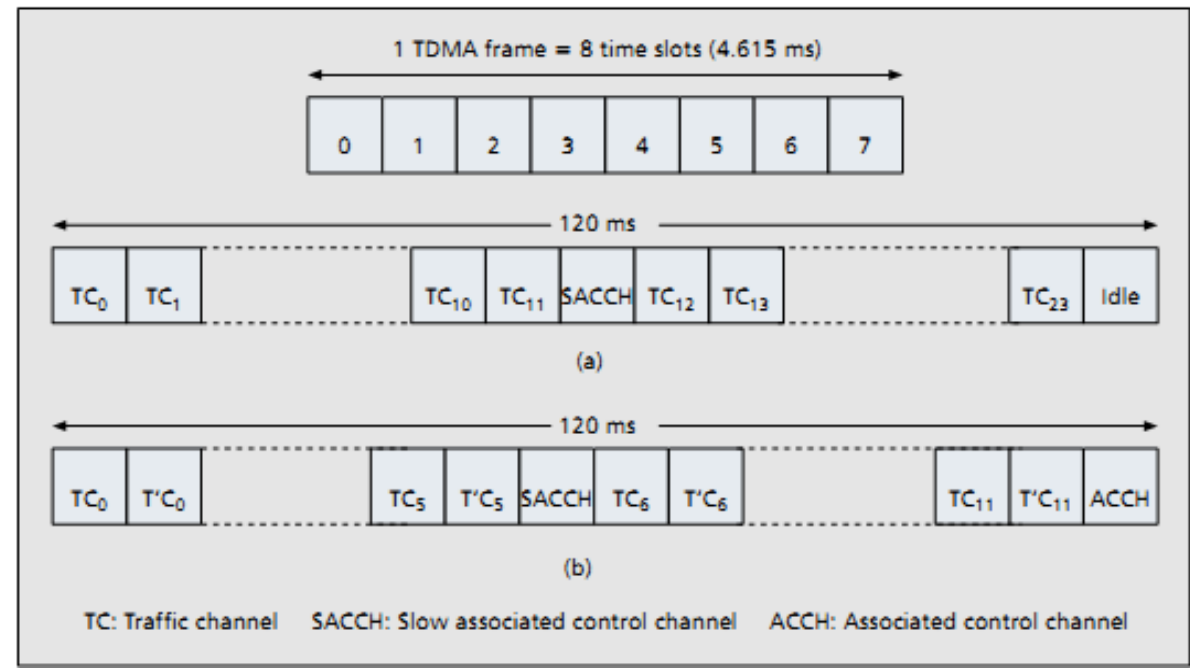

Figure 1: Full-rate and half-rate channels in GSM system: a) one full-rate channel; b) two half-rate channels

Multiple access in telecommunications systems refers to techniques that enable multiple users to share limited network resources efficiently. A telecommunications network has finite resources that are usually defined in terms of bandwidth. When there is more than one user to access such limited bandwidth, an MA scheme must be put in place to control the share of bandwidth among multiple users so that everyone can use services provided by the network and to make sure that no single user spends all available resources.

\section{REQUIREMENTS FOR A MULTIPLE ACCESS SCHEME}

There are a number of requirements that any multiple access scheme must be able to meet:

- Ability to handle several users without mutual interference.

- Ability to be able to maximize the spectrum efficiency

- Must be robust, enabling ease of handover between cells.

From a very early stage of modern communications, researchers have been working on finding the best MA scheme to follow the above simple rule of resource sharing among multiple users. Very visible and fundamental ways of sharing bandwidth, frequency and time separation, were chosen as the beginning of MA generation. In the first MA communications systems, frequency modulation (FM) could divide the available frequency spectrum for a given system into some frequency channels where each channel occupies a portion of total available bandwidth and is given to a single user. Multiple users using separate frequency channels could access the same system without significant interference from other users concurrently operating in the system. It is the simplest way of having an MA scheme in a multi-user system, and it is referred to as frequency-division MA (FDMA).

With the same concept, time-division MA (TDMA) schemes came to start the digital communications era by dividing the time axis into portions or time slots, each assigned to a single user to transmit data information. TDMA schemes thus came into effect through frame and multiframe concepts: a user could send a large data file within time slots of periodical frames. Data from a single user always sits in the same time slot position of a frame, so at the receiver all information from that portion can be collected and aggregated to shape the original transmitted packet. TDMA, together with pulse code modulation (PCM), has become an effective way of sharing the available system resources not only in wireless communications but in wired communications since then. TDMA has kept its dominance in wired and wireless systems for many years. Many cellular standards such as the second-generation (2G) Global System for Mobile Communications (GSM) and the 2.5G General Packet Radio Service (GPRS) adopted TDMA as their MA scheme.

As it is clear from the above simple review, in both FDMA and TDMA techniques the number of channels or time slots is fixed for a given system, and a single channel is allocated to a single user for the whole period of communications. This was not only a concept to have a simple MA technique in the early stage of modern telecommunications, but was based on the dominant service in mind at the time, voice communications. Having a fixed channel or time slot assignment could guarantee the service quality for real-time and constantbit-rate voice telephony, the main service at that time. By increasing the number of services from simple voice to more burst data transmissions, fixed channel assignment has shown its lack of efficiency in utilizing the scarce spectrum, especially with the exponential increase in number of users. 


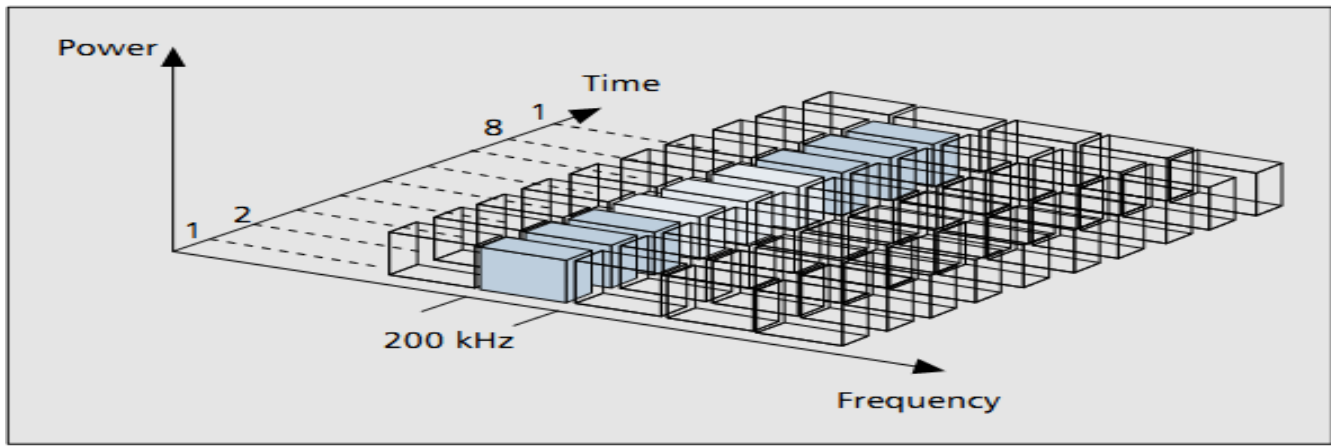

Figure 2: Physical channels in a GPRS system

Researchers started to think of more dynamic channel assignment forms of TDMA and FDMA that could allocate a channel only when the user wants to transmit data. While many dynamic channel assignment MA schemes have been invented since then, the fixed upper limit on number of users in a TDMA or FDMA system has created a demand for new MA schemes with fewer limitations, particularly for mobile communications.

With this idea in mind, code-division multiple access (CDMA) schemes based on spread spectrum technology started to come into commercial systems, different from their original environment mainly in military applications. In a CDMA system the relatively narrowband user's information is spread into a much wider spectrum using a high clock (chip) rate. Using different uncorrelated codes by each user, it is possible to send multiple users' information on the same frequency spectrum without significant difficulty in detecting the desired signal at the receiver side as long as the correct spreading code is known to the receiver. The signal from each user will have every low power and be seen by others as back-ground noise. Therefore, as long as the total power of noise (i.e., multi-user interference) is less than a threshold, it is possible to detect the desired signal using the spreading code used to encode the signal at the transmitter. Using spread spectrum techniques, CDMA has become a dynamic channel allocation MA scheme that has no rigid channel allocation limitation for individual users. The number of users is also not fixed as in TDMA and FDMA, and a new user can be added to the system at any time. The upper limit for the maximum number of simultaneous users in the system using the same frequency spectrum is decided by the effect of total power of multi-user interference; thus, adding new users to a CDMA system will only cause graceful degradation of signal quality. CDMA is thus seen as an MA scheme that has no fixed maximum number of users as opposed to TDMA and FDMA schemes.

With the exponential increase in the number of users for mobile cellular communications and the development of $3 \mathrm{G}$ wireless cellular systems, CDMA, with its proven capacity enhancement over TDMA and FDMA, has been chosen as the main MA scheme for 3Gmobile cellular systems.

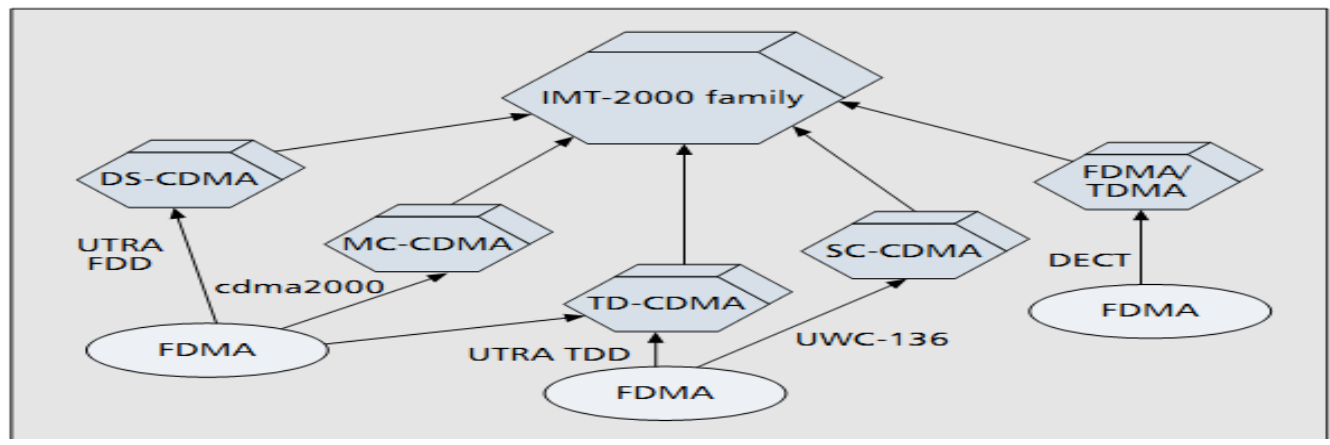

Figure 3: The IMT 2000 family of radio access technologies

International Mobile Telecommunications (IMT-2000) as the standard body within the International Telecommunication Union (ITU) has identified five radio access technologies for 3G, as shown in Figure 3. As illustrated in this figure, four out of five systems use pure CDMA or a CDMA combination in IMT-2000.

MA schemes chosen for $3 \mathrm{G}$ systems could direct us to identify the requirements for MA schemes to be developed in B3G systems. Using the example of Universal Mobile Telecommunication System (UMTS), we can see two modes of operation in the UMTS terrestrial radio access (UTRA): time-division duplex (UTRATDD) and frequency-division duplex (UTRA-FDD). The two modes can be considered a combination of CDMA with FDMA and TDMA, respectively, by separating frequency spectrum for downlink and uplink in UTRA-FDD and using the same spectrum for both uplink and downlink channels in UTR-TDD. 
Despite having two different modes of operation in the UMTS air interface, some basic system parameters such as carrier spacing, chip rate, and frame length are used similarly in order to have possible harmonization in UMTS. This would allow dual mode FDD/TDD terminals and internetworking for UMTS users. UTRA-FDD is purely a CDMA based system where user signals are differentiated from one another by power and the code they use. UTRA-TDD, on the other hand, is a kind of TDMA plus CDMA where user signals are differentiated not only by their power and code but also by their time slot allocations.

Having two modes of operation for a single UMTS system provides flexibility in handling adverse multimedia services and also different traffic densities in the network. With TDD it is possible to flexibly arrange different numbers of time slots on uplinks and downlinks in accordance with traffic conditions. In modern communications systems with more Internet-based traffic loads, it is necessary (in usual cases) to give more capacity to the downlink than to the uplink (e.g., when browsing the Internet). Frequency division fails to have such flexibility as the channels on uplinks and downlinks are separated in the beginning. However, TDD can change the ratio of uplink and downlink capacity based on dynamic requirements of the network at any time. This concept would be necessary for B3G systems where burst data traffic becomes the dominant part of the network load.

For a similar reason, TDD could work better in smaller cell sized networks than its FDD counterpart. Therefore, the TDD mode will be more efficient in pico and microcellular (indoor) cases, while FDD will work more efficiently in macrocellular outdoor situations. TDD could manage dense traffic in urban areas much better than FDD due to its choice of allocation of timeslots on uplink and downlink depending on traffic conditions. A combination of the two modes considered in UMTS is capable of delivering data and voice traffic in adverse channel and cellular conditions. Also, a combination of different MA schemes on uplink and downlink could provide further flexibility in spectrum management in a cellular network. We will continue to address the features of MA schemes in 3G cellular systems.

\section{CELLULAR GENERATION}

$1 G$ - Analog

Introduced in the late 1970s and early 1980s, the first cellular systems were analog. They were used for data just like land-based telephone lines are used for dial-up with analog modems. A handful of cell phone models could be adapted to laptop modems and transfer data at less than $15 \mathrm{Kbps}$ while traveling. (e.g. AMPS, TACS and NMT.)

\section{G - 2.5G - Digital}

The second generation refers to digital voice cell phone systems deployed in the 1990s, which were based on GSM, TDMA or CDMA. Several so-called 2.5G (or $2 \mathrm{G}+$ ) technologies added data services for Internet access and e-mail with typical downstream speeds ranging from 64 to $200 \mathrm{Kbps}$ for the user. These include GPRS, EDGE and IS-95B. (e.g. GPRS, EDGE and IS-95.)

\section{G - High Speed}

Launched after the turn of the century, the third generation offers faster access to the Internet and other data services with typical downstream speeds ranging from $400 \mathrm{Kbps}$ up to $1 \mathrm{Mbps}$ for the user. Defined by the ITU under the IMT-2000 framework, 3G is implemented regionally in Europe (UMTS), North America (CDMA2000) and Japan (NTT DoCoMo). EV-DO, WCDMA and HSDPA are examples of 3G data services. 3G also embraces worldwide roaming for global travelers. (e.g. GAN, EV-DO, WCDMA, HSDPA, CDMA2000, UMTS and 3GPP.)

\section{G - Higher Speed for Movies and TV}

In the 2008-2010 time frame, $4 \mathrm{G}$ cell phones are expected to support TV in realtime as well as video downloads at high speed. It is also expected to embrace automatic roaming to non-cellular systems such as Wi$\mathrm{Fi}$, satellite and other wireless networks, whichever is most appropriate. NTT Docomo's 4G service is based on WCDMA; however, carriers in the U.S. and Europe are expected to deploy other air interfaces. (E.g. NTT DoCoMo and WCDMA.

\section{MATHEMATICAL REPRESENTATION OF MULTIPLE ACCESS TECHNOLOGIES}

In this section we explain different MA schemes using simple mathematical expressions that make it easy to understand the main features of MA schemes. This discussion could also be used for future implementation of MA schemes in B3G wireless cellular systems. $\mathrm{PT}_{\mathrm{b}}\left(\mathrm{t}-\mathrm{T}_{\mathrm{b}}\right)$ Generally, the transmitting signal of a user $k$ can be expressed as $s_{k}(t)=2 \sqrt{P_{k}(t)} b_{k}(t) \cos \omega_{c} t$, where $a_{k}(t)$ is an MA function, $b_{k}(t)$ is the data signal of user $\mathrm{k}$, and $\omega_{\mathrm{c}}$ is a carrier angle frequency. For the sake of simplicity and without lack of generality, we consider 
binary phase shift keying (BPSK) modulation and assume that every user transmits with a fix data rate, $\mathrm{R}_{\mathrm{b}}$ $=1 / T_{b}$, where $\mathrm{Tb}$ is the bit duration in seconds. The data signal, $\mathrm{b}_{\mathrm{k}}(\mathrm{t})$, is given as

$$
\mathrm{bk}(\mathrm{t})=\sum_{\mathrm{m}=-\infty}^{\infty} \mathrm{bm}, \mathrm{kPTb}(\mathrm{t}-\mathrm{Tb})
$$

where $b_{m, k} \in\{-1,1\}$ is the $m^{\text {th }}$ bit data of user $k$ and $p_{x}(t)$ is a pulse wave function equal to 1 for $0 \leq t<x$, and 0 otherwise.

The choice of $a_{k}(t)$ is very important in designing an MA scheme. For $2 \mathrm{G}$ and $3 \mathrm{G}$ systems, the design criterion is to minimize the cross-correlation between users such that $\left.J_{T b} a_{k}(t) a_{k^{\prime}(\neq k}\right)(t)<\varepsilon$, where $\varepsilon$ is the cross-correlation factor.

As explained earlier, in principle there are two MA functions: time domain and frequency domain. We show, in the following, that those two MA functions can be expressed in the form of CDMA. Furthermore, the signal-to-noise-plus-interference ratio (SNIR) of time-and frequency-domain MA functions are the same.

\subsection{Time-Domain Multiple Access}

DS-CDMA - For DS-CDMA, the MA function is given as

$\operatorname{ak}(\mathrm{t})=\sum_{\mathrm{l}=-\infty}^{\infty} \operatorname{aklPTc}(\mathrm{t}-\mathrm{lTb})$

where $a_{k}{ }^{1} \in\{-1,1\}$ is the $1^{\text {th }}$ signature sequence of user $k$. The signature sequence $a k=\left\{a_{k}{ }^{1}, a_{k}{ }^{2}, \ldots, a_{k}{ }^{N}\right\}$ has a period of $\mathrm{N}=\mathrm{T}_{\mathrm{b}} / \mathrm{T}_{\mathrm{c}}$ ( $\mathrm{T}_{\mathrm{c}}$ is the chip duration). In most cases, signature sequences with $\varepsilon \cong 0$ are used.

The special case of signature sequence is that of orthogonal sequences ( i.e., $\varepsilon=0$ ). This can be achieved by either time-synchronous CDMA or TDMA.

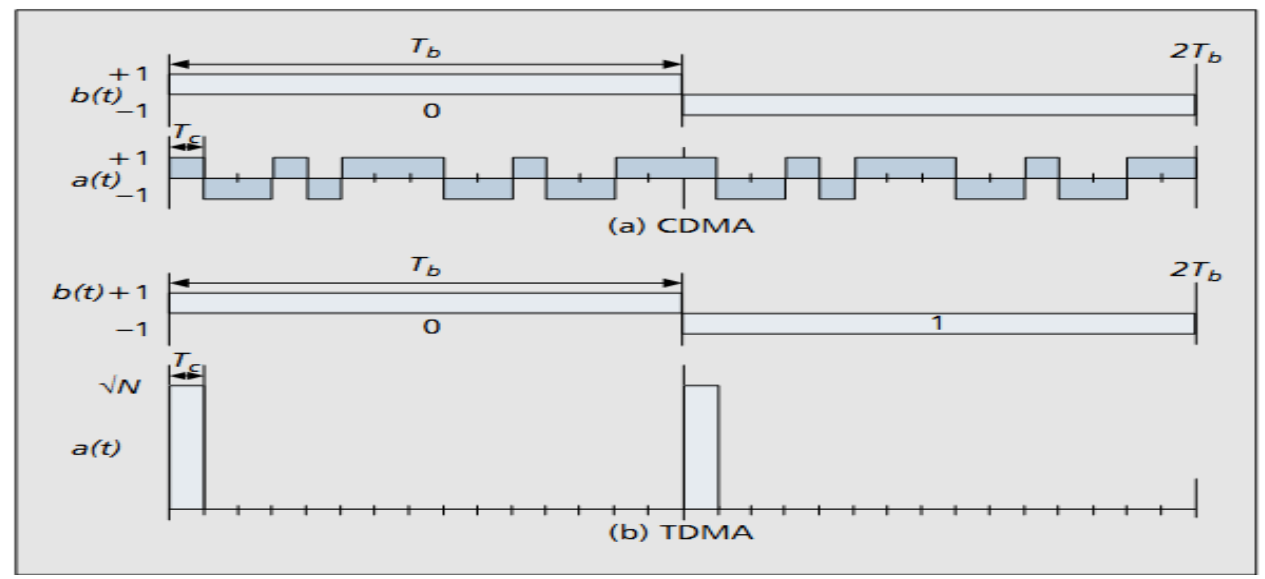

Figure 4: An example of the signature sequence for time domain multiple access: a) CDMA; b) TDMA

TDMA - In TDMA a sequence with only one element is used. For example, $\mathrm{a}_{1}=\{\mathrm{N}, 0, \ldots, 0\}, \mathrm{a}_{2}=\{0, \mathrm{~N}, 0, \ldots$ $0\}, \ldots$ Examples of signature sequences of CDMA and TDMA systems are depicted in Fig. 4.

\subsection{Frequency-Domain Multiple Access}

Multicarrier CDMA (MC-CDMA) - Now we consider the MA function given as

$$
\operatorname{ak}(\mathrm{t})=\sum_{\mathrm{l}=1}^{\mathrm{Nf}}(\sqrt{2 \mathrm{P})} \text { aklcos } \omega \mathrm{lt}
$$

where $\mathrm{N}_{\mathrm{f}}$ is the spreading factor for the case of frequency domain spreading. The spreading sequence designed for DS-CDMA can be applied in this case too. The design criterion is also the same. With a smaller $\varepsilon$, better MA performance is achievable. We note that if the total bandwidth and data rate are the same for timeand frequency-domain spreading schemes, we have the following relations [4]: $\left(1+\mathrm{N}_{\mathrm{f}}\right) / \mathrm{T}_{\mathrm{b}}=(1+\alpha) / \mathrm{T}_{\mathrm{c}}$ and $1+$ $\mathrm{N}_{\mathrm{f}}=(1+\alpha) \mathrm{N}$, where $\alpha$ is the roll off factor of the root cosine roll off filter. We introduce this band limitation to DS-CDMA in order to achieve the same spreading factor. If we set $\alpha=0$, we have the same spreading factor for both DS- and MC-CDMA schemes. 


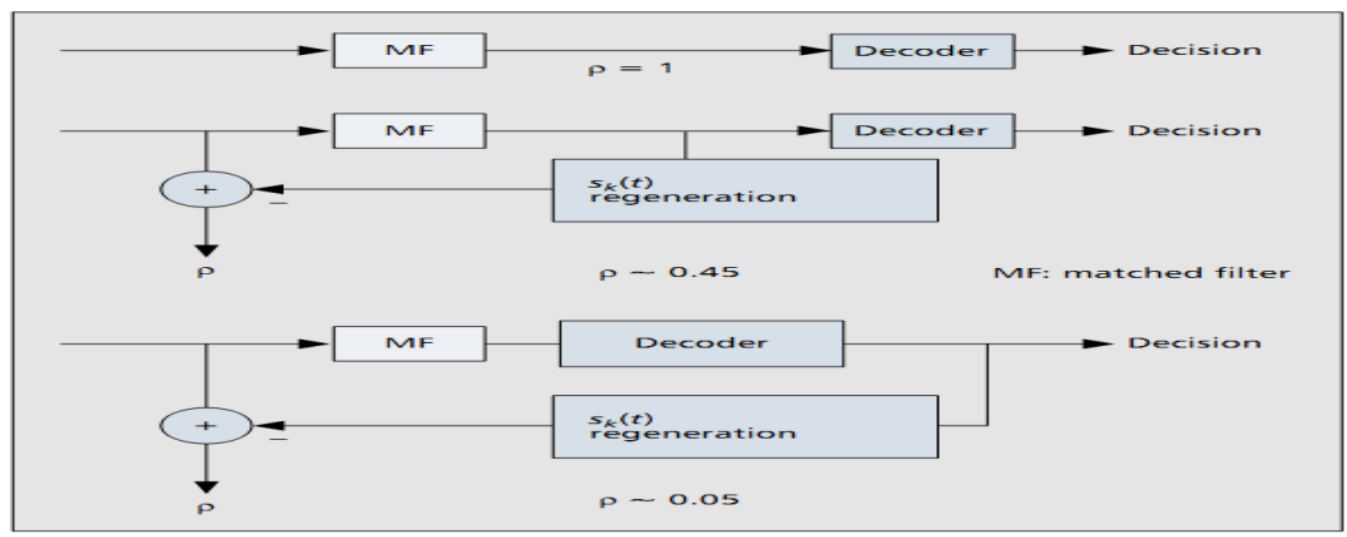

Figure 5: Interference cancellation factor

FDMA - FDMA is a special case of MC-CDMA. If we design the signature sequence with only one element and avoid spectrum over-lap, $\varepsilon=0$ can be realized. In this case the sequences are, for example, $a_{1}=\left\{N_{f}, 0, \ldots\right.$, $0\}, \mathrm{a}_{2}=\left\{0,0, \mathrm{~N}_{\mathrm{f}}, 0, \ldots, 0\right\}, \ldots$

\section{SNIR}

Signal-to-interference-plus-noise ratio is given as

$\mathrm{SNIR} \approx 1 /\left[\rho \in(\mathrm{K}-1)+\mathrm{N}_{0} / 2 \mathrm{E}_{\mathrm{b}}\right]$,

where $E_{b}$ is the bit energy and N0 is the power spectrum of background noise. The (MA) interference terms for different MA schemes are:

- For TDMA and FDMA: $\varepsilon=0$

- For DS-CDMA: $\varepsilon=(1-\alpha / 4)$

- For MC-CDMA: $\varepsilon=1 / 2 \mathrm{~N}_{\mathrm{f}}$

If we set $\alpha=0$ for DS-CDMA, we have the same SNIR for both DS- and MC-CDMA.

Interference cancellation or multi-user detection can enhance performance with a reasonable trade-off between performance and complexity. The term $\rho$ is the average interference cancellation factor, which we define as the ratio between the average remaining interference power after cancellation and the total interference power without cancellation. This interference cancellation is mandatory and may play an important role in designing an MA scheme, especially for $3 \mathrm{G}$ and $\mathrm{B} 3 \mathrm{G}$ cellular systems.

In Fig. 5 examples of two main cancellation techniques are shown with some typical values of $\rho$. If no cancellation is performed, we have $\rho=1$. If the feedback soft value is generated after detection, we have $\rho \cong$ 0.45 . If the error correcting decoder is used for feedback soft value regeneration, we have $\rho \cong 0.05$.

The importance of the cancellation technique with a small $\rho$ is that it allows a rather large value of $\varepsilon$. This means we have more freedom to design the MA scheme.

Spectral efficiency is defined as

\section{SPECTRAL EFFICIENCY}

$\eta=\left(K / W_{\text {sys }}\right) \cdot(1 / \mathrm{AC}) \cdot \mathrm{G}\left[\mathrm{Erlang} / \mathrm{m}^{2} / \mathrm{Hz}\right]$,

where $\mathrm{W}_{\text {sys }}[\mathrm{Hz}]$ is the total system bandwidth, $\mathrm{A}\left[\mathrm{m}^{2}\right]$ is the total area per cell, $\mathrm{C}$ is the cell frequency reuse factor, and $\mathrm{G}$ [Erlang/channel] is the offered traffic per channel [6]. For TDMA and FDMA, $\mathrm{C}=3$ or $\mathrm{C}=$ 7 may be typical values, and $\mathrm{C}=1$ is the case for CDMA. The total number of simultaneous users in the system, $\mathrm{K}$, is then obtained for a given SNIR to achieve the required level of quality of service (QoS).

We note that the first factor in the spectral efficiency expression, $\mathrm{K} / \mathrm{W}_{\mathrm{sy}}$, corresponds to the bandwidth efficiency, which can be enhanced by bandwidth efficient modulation such as multiple-input-multiple-output (MIMO) or orthogonal frequency-division multiplexing (OFDM). The second factor, 1/AC, corresponds to the spatial efficiency. If the coverage of a base station can be reduced, spatial efficiency can be improved. Smart antenna techniques and antenna diversity can also enhance spatial efficiency. With a base station having several remote antennas, it is also possible to enhance efficiency. If we consider multihop cellular systems, higher spatial efficiency can be realized.

The final factor, G, is network efficiency. This can be enhanced by employing a voice activity factor or appropriate multimedia transmission so that appropriate traffic balance can be maintained over separate channels. However, if the system is composed of different network configurations, some reduction in efficiency may occur due to the restrictions from the network configuration. Multihop cellular systems are examples of such network configurations where a remote user could be accessed through other users residing in the coverage area of network base stations. 


\section{KEY FEATURES OF 3G MULTIPLE ACCESS TECHNOLOGIES}

B3G mobile systems will follow the footpath of $3 \mathrm{G}$ systems; therefore, it is important to address key features of $3 \mathrm{G}$ systems in order to have a good start on the development of the new system. In this section we address those features in $3 \mathrm{G}$ mobile systems.

The IMT-2000 terrestrial radio interface has five standards, four of which are based on CDMA (Fig. 3). This indicates that CDMA is a quite promising access technology for high-data-rate wireless communications. Three typical CDMA-based MA schemes receiving attention in IMT-2000 are DS-CDMA, MC-CDMA, and time-division CDMA (TD-CDMA).

CDMA schemes have some impressive advantages that TDMA and FDMA do not have. One is tolerance to the effects of channel fading. In DS-CDMA we can apply a RAKE diversity technique that can improve performance against severe multipath fading channels. Besides, in MC-CDMA guard band intervals can mitigate the effect of multipath fading. An advantage in cellular mobile systems based on CDMA is that we can achieve efficient frequency reuse. Because users are distinguished by their own codes, every cell can use the same frequency (i.e., $\mathrm{C}=1$ in the spectral efficiency expression); therefore, we can obtain higher frequency efficiency. Additionally, soft handover among cells is achievable.

We should, however, notice some requirements for utilizing CDMA. A considerable problem in CDMA is interference from other users. A value of cross-correlation $\varepsilon$ is usually non-zero in CDMA, and it limits channel capacity. To improve channel capacity, we must consider how to mitigate the effects of multiuser interference. According to the discussion of SNIR given in the previous section, we can reduce the interference by decreasing $\varepsilon$ and/or the interference cancellation factor $\rho$. In practical situations we usually introduce interference cancellation or multi-user detection in order to decrease $\rho$. Besides, inorder to reduce the cross-correlation factor $\varepsilon$, we utilize suitable spreading code sequences that have small cross-correlation. By synchronous or quasi-synchronous CDMA, we can also reduce interference from other users .

In cellular mobile systems with CDMA, we face the near-far problem too. A signal transmitted by a user who is far from the base station can easily be blocked by a signal from a nearby user. To solve the near-far problem, we should therefore introduce a power control technique in CDMA systems to maintain the quality of signals from far users. Thus, the base station should frequently send power control information to every user.

MC-CDMA has several advantages over DS-CDMA. One advantage of MC-CDMA is that it is suitable for frequency selective fading channels. As mentioned, DS-CDMA needs a RAKE receiver, which may increase the complexity of the receiver. MC-CDMA, on the other hand, can compensate for fading by using frequency equalization in the frequency domain, resulting in lower complexity. Another advantage of $\mathrm{MC}$ CDMA is its low interference and less outband radiation. The spreading factor of DS-CDMA is $N\left(=\mathrm{T}_{b} / \mathrm{T}_{\mathrm{c}}\right)$. From the discussion given in the previous section, the spreading factor for MC-CDMA, in contrast, is $\mathrm{N}(1+\alpha)-$ 1 with the same bandwidth and data rate. A larger spreading factor in MC-CDMA is therefore available in order to reduce the effect of user interference. In MC-CDMA, however, since a signal is composed of subcarriers, its amplitude has large fluctuations. Thus, we require a high linear amplifier that causes inefficient power consumption.

TD-CDMA can compensate for a disadvantage of CDMA. As mentioned earlier, in CDMA-based schemes a power control technique is required, and therefore power control information should be communicated between mobile users and base stations. TD-CDMA is appropriate to share the information because it uses the same channel on both uplink and down-link. It is also easy to share channel information between two stations. Another advantage of TD-CDMA is that it achieves efficient asymmetric communications between uplink and downlink. We can assign each time slot to uplink or down-link according to the required bit rates. In TD-CDMA time delay would also occur. Furthermore, a long overhead should be required for synchronization. We also need to consider mitigating the effect of blocking a signal from other users in the asymmetric mode in TD-CDMA systems.

Before closing this section, we should mention another important MA scheme. Space-division multiple access (SDMA) is a scheme that can provide high channel capacity in mobile cellular systems. In SDMA, as its name indicates, users are separated in a spatial way, which is very different from the MA schemes discussed earlier. In this scheme generally an adaptive array antenna technique is adopted. The adaptive array antenna can make the beam pattern flexible as needed, and therefore it is possible to make each suitable beam pattern correspond to one user. A system with L array antennas can generally distinguish at most L users. One remarkable advantage is that every user can share the same channel resource such as frequency and/or time slot. This property suggests that SDMA can easily enhance channel capacity by collaborating with other MA schemes such as FDMA, TDMA, and CDMA. One disadvantage of SDMA is that the MA gain is considerably influenced by the location of users. We face the difficulty of separating two users who are placed near the base station. The other problem is the complexity of hardware for tracking the signals. The mobile terminal continuously and some-times rapidly changes its location. In order to keep a high carrier-to-interference ratio (CIR), there is a need for an accurate and rapid tracking algorithm. In SDMA, in addition to intercell handover, 
we have to consider an internal hand-over technique, which will occur when the beams from two users get close and finally cross over each other.

\section{MULTIPLE ACCESS TECHNOLOGIES FOR B3G SYSTEMS}

In this section we describe the main features required for MA schemes in B3G systems and address promising techniques. In B3G mobile systems, high-date-rate transmission on the order of $10 \mathrm{Mb} / \mathrm{s}$ and more is expected. With the existing MA schemes, several technical issues prevent us from achieving such a high transmission rate. In DS-CDMA, many weak multipaths tend to appear. Because of the large number of weak peaks, we face the difficulty of achieving coherent RAKE combination and consequently cannot obtain enough diversity from RAKE diversity. In contrast, since the transmission rate on MC-CDMA channels is slower than that of DS-CDMA, the influence of multipath tends to mitigate high-speed communications. However, on uplinks utilizing MC-CDMA, transmitting a signal from each user is affected by its own fading, and each subchannel has also its own fading effect. It is difficult to introduce an actual compensation method for the fading effect of every user and every subchannel. Although CDMA is essentially capable of becoming the MA technique for $\mathrm{B} 3 \mathrm{G}$, the current CDMA schemes unfortunately do not satisfy the requirements of B3G. Multicarrier DS-CDMA (MC/DS-CDMA) scheme has received much attention as one promising MA scheme for B3G systems. Because MC/DS-CDMA can be explained as a combination of MC-CDMA and DS-CDMA, MC/DS-CDMA achieves very-high-data-rate transmissions with the advantages of both schemes. For example, we can obtain the RAKE diversity effect even at high-data-rate transmissions and improve performance in multipath fading environment. Furthermore, similar to MC-CDMA, MC/DS-CDMA is robust against frequency selective fading. One impressive advantage of MC/DS-CDMA is the flexibility of assigning spreading codes to two domains (i.e., time and frequency). In order to obtain a certain processing gain in DS-CDMA, we have to decide the chip rate according to the processing gain. Similarly, the processing gain determines the number of subcarriers in MC-CDMA. In contrast with both schemes, in MC/DS-CDMA we have the freedom in decision of such parameters according to the fading effect, system requirements, and so on. Of course, MC/DS-CDMA has some disadvantages with respect to DS-CDMA and MC-CDMA. For example, similar to MC-CDMA, MC/DS-CDMA requires an amplifier with high linearity, which results in power inefficiency.

TDD-CDMA is a modified version of the 3G TD-CDMA introduced in [8] as an attractive scheme for B3G systems. An important advantage of TDD-CDMA is that it has better frequency efficiency than UTRAFDD. Using FDD mode, a large frequency guard band is needed that makes inefficient use of frequency spectrum. Similar to TD-CDMA, it is possible to share the channel information between base station and mobile terminals in TDD-CDMA. The advantage is particularly more visible in high-data-rate transmissions because of rapid changes in the fading environment. In B3G systems the requirement for bursty data communications will increase, which will result in a significant increase in downlink traffic load. TDD provides enough capability to satisfy such a requirement because it can easily achieve asymmetric trans-missions; therefore, TDD-CDMA will become a major MA scheme for B3G systems as an evolved version of TD-CDMA already used in 3G systems. Finally, orthogonal frequency-division MA (OFDMA) has attracted much attention, particularly in the emerging IEEE 802.16 standard, as the last mile for wireless communications. The concept of OFDMA is essentially the same as FDMA, but it has some advantages not included in FDMA. In OFDMA, every user shares the fast Fourier transform (FFT) space for uplink, and the base station can assign subcarriers to users. Thus, transmission with different rates among users is available according to each user's requirement. On frequencyselective channels, however, performance of a user assigned by a deep faded subcarrier is damaged by fading. To avoid the effect of fading, we usually introduce a frequency hopping (FH) technique with forward error correction (FEC). Furthermore, since OFDMA is a quasi-synchronous MA scheme, a large guard time that compensates for an access delay is required.

\section{APPLICATIONS}

All the above multiple access technologies are meant for wireless communication for a variety of applications such as interactive multimedia, voice over IP, network games, videoconferencing, mobile networks, personal communication, message service etc..

\section{ECONOMIES/MAJOR INDUSTRIES}

With over 900 million wireless subscribers, India is one of the biggest markets in the telecom industry globally. From GSM, CDMA and even broadband subscribers, the overall penetration in India is nearly 75 percent. Here is a list of the top 5 Indian telecom companies based on their market share.

1. Airtel functions in 20 countries and has its operations spread across South Asia, Africa and the Channel Islands. It offers services like $2 \mathrm{G}, 3 \mathrm{G}$ and $4 \mathrm{G}$ depending upon the country of operation. It is the fifth largest telecom operator in the world. 
2. RCOM is the world's 16th largest mobile phone operator and was founded in the year 2004. It provides both $2 \mathrm{G}$ and $3 \mathrm{G}$ services across the nation. The Reliance group can be credited for the telecom revolution in the country

3. Vodafone India, formerly known as Vodafone Essar and Hutchison Essar was Founded in the year 1994. It is based in Mumbai, Maharashtra. It provides both $2 \mathrm{G}$ and $3 \mathrm{G}$ services across the nation. Its advertising campaign of ZooZoos became a rage, and has given the brand a lot of recognition and respect

4. Idea Cellular is part of the Aditya Birla Group, with its headquarters situated in Mumbai, India. It was founded in the year 1995. The company with its "Idea" branding has been able to create a huge market

5. BSNL is a state-owned telecom company. Its headquarters are situated in New Delhi, India. It is the largest provider of fixed telephony and fourth largest mobile telephony provider in India. It even provides broadband services.

\section{CONCLUSIONS}

In this article state-of-the art technologies for MA schemes that have been adopted in $3 \mathrm{G}$ wire-less cellular systems are examined. We provide a novel mathematical expression that could inter-connect all these MA schemes usually separated under time division, frequency-division, and code-division techniques. Several combinations of MA schemes are discussed in the article, addressing their application in different cellular mobile standards. Based on the summary of features of individual MA schemes and the requirements for B3G systems in terms of high-data-rate transmission and burst data traffic network load, we conclude that none of the existing MA techniques would be able to satisfy the severe transmission criteria in B3G systems. Combination of different MA schemes, use of MA schemes in an appropriate way for different environments, use of different MA schemes on downlinks and uplinks of cellular networks, with proper scheduling of multimedia traffic and MA scheme would be the ultimate way to solve the problem of fair spectrum allocation in multi-user environments. Next-generation networks will be developed through good management and a combination of the advanced multiple access technologies discussed in this article, rather than the development of new schemes.

\section{REFERENCES}

[1]. L. Bos and S. Leroy, "Toward an all-IP UMTS System Architecture," IEEE Network, vol. 15, no. 1, 2001, pp. 36-45.

[2]. S. G. Glisic, Advanced Wireless Communications - 4G Technologies, Wiley, 2004.

[3]. Jamalipour, The Wireless Mobile Internet - Architectures, Protocols and Services, Wiley, 2003.

[4]. H. Muramatsu et al., "Effect of Nonlinear Amplifiers of Transmitters in Multicarrier CDMA Systems," IEICE Trans. Fundamentals, vol. J85-A, no. 3, Mar. 2002, pp. 340-48.

[5]. Z. Dawy and A. Seeger, "Coverage and Capacity Enhancement of Multiservice WCDMA Cellular Systems via Serial Interference Cancellation,” Proc. ICC 2004,Paris, France, June 2004.

[6]. G. L. Stuber, Principle of Mobile Communications, Kluwer, 1996.

[7]. D. Yu, H. Li, and H. Hagenauer, "Multihop Network Capacity Estimation," Proc. ICC 2004, Paris, France, June 2004.

[8]. R.Esmailzadeh and M. Nakagawa, "TDD-CDMA for the 4th Generation of Wireless Communications," IEEE Commun. Mag., vol. 41, no. 8, Aug. 2003, pp. 8-15.

[9]. F. Piolini and A. Rlolando, "Smart Channel-Assignment Algorithm for SDMA Systems," IEEE Trans. Microwave Theory and Tech., vol. 47, no. 6, June 1999, pp. 693-99.

[10]. S. Suwa, H. Atarashi, and M. Sawahashi, "Performance Comparison between MC/DS-CDMA and MC-CDMA for Reverse Link Broadband Packet Wireless Access," Proc. VTC-2002 Fall, Vancouver, Canada, Sept. 2002, pp. 2076-80. 